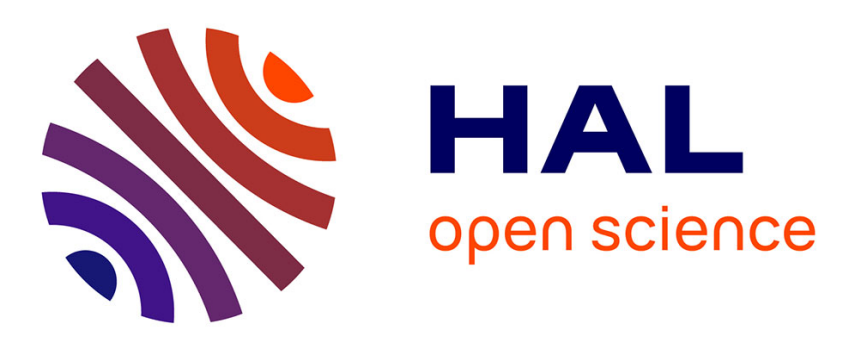

\title{
Bayesian Statistical Identification of Orthotropic Elastic Constants Accounting for Measurement and Modeling Errors
}

Christian Gogu, Raphael T. Haftka, Rodolphe Le Riche, Jérôme Molimard, Alain Vautrin, Bhavani V. Sankar

\section{To cite this version:}

Christian Gogu, Raphael T. Haftka, Rodolphe Le Riche, Jérôme Molimard, Alain Vautrin, et al.. Bayesian Statistical Identification of Orthotropic Elastic Constants Accounting for Measurement and Modeling Errors. 11th AIAA Non-Deterministic Approaches Conference, May 2009, Palm Springs, United States. pp.No. AIAA-2009-2258. emse-00686558

\section{HAL Id: emse-00686558 https://hal-emse.ccsd.cnrs.fr/emse-00686558}

Submitted on 14 Jan 2019

HAL is a multi-disciplinary open access archive for the deposit and dissemination of scientific research documents, whether they are published or not. The documents may come from teaching and research institutions in France or abroad, or from public or private research centers.
L'archive ouverte pluridisciplinaire HAL, est destinée au dépôt et à la diffusion de documents scientifiques de niveau recherche, publiés ou non, émanant des établissements d'enseignement et de recherche français ou étrangers, des laboratoires publics ou privés. 


\title{
Bayesian Statistical Identification of Orthotropic Elastic Constants Accounting for Measurement and Modeling Errors
}

\author{
Christian Gogu ${ }^{1, a, b}$, Raphael T. Haftka ${ }^{2, b}$, Rodolphe Le Riche, ${ }^{3, a}$, Jérôme Molimard ${ }^{4, a}$, Alain Vautrin ${ }^{5, a}$, Bhavani V. \\ Sankar ${ }^{6, b}$ \\ ${ }^{a}$ Centre Science des Matériaux et des Structures, Ecole des Mines de Saint Etienne \\ 158 cours Fauriel, 42023 Saint Etienne cedex 2, France \\ ${ }^{b}$ Department of Mechanical and Aerospace Engineering, University of Florida
}

PO Box 116250, Gainesville, FL 32611-6250, USA

$11^{\text {th }}$ AIAA Non-Deterministic Approaches Conference

4 - 7 May 2009, Palm Springs, California

\begin{abstract}
Bayesian identification provides a framework that can handle both measurement and modeling errors. Furthermore it identifies a probability distribution function thus providing information on both variance and correlation of the identified properties. However, the procedure can be very costly computationally. In order to address the computational cost issue a Bayesian identification procedure based on response surface methodology is proposed. The procedure is illustrated on the problem of identifying orthotropic elastic constants from natural frequencies of a free composite plate. The procedure accounts for measurement noise, uncertainty in other input parameters to the vibration model (plate dimensions, density) as well as systematic error effects. The joint probability distribution of the four elastic ply constants is identified and characterized by mean value and variancecovariance matrix. We find that some properties, such as Poisson's ratio, are identified with much higher uncertainty than other and that significant correlation between the identified properties is present. The developed procedure allowed substantial reduction in computational cost. However, in spite of the cost reduction techniques, it remains at the edge of what is presently reasonable computation time.
\end{abstract}

\section{Introduction}

Accurate determination of the orthotropic mechanical properties of a composite material has always been a challenge to the composites community. This challenge is threefold: designing the most appropriate experiment for determining the properties sought, handling inherent uncertainties in the experiment and modeling of the experiment and finally estimating and controlling the uncertainty with which the properties are determined.

The design of an experiment for identifying the four in-plane elastic constants of an orthotropic material as accurately as possible has been a rich area of investigation (e.g. [1]-[5]). Vibration testing is considered as an effective experimental method for this purpose. This technique was introduced in [6]-[8] in the context of determining the elastic constants of a composite. These studies involved measuring the natural frequencies of a

\footnotetext{
${ }^{1}$ Graduate Research Assistant, AIAA Student Member, Corresponding Author (christian.gogu@gmail.com)

${ }^{2}$ Distinguished Professor, AIAA Fellow

${ }^{3}$ Chargé de Recherche CNRS

${ }^{4}$ Associate Professor

${ }^{5}$ Professor

${ }^{6}$ Ebaugh Professor, AIAA Associate Fellow
} 
freely hanging composite plate, frequencies which were used for identifying the four elastic constants of the laminate using model updating. Some of the advantages of vibration testing outlined in [6]-[10] are single test of nondestructive nature and determination of homogenized properties as opposed to local properties using strain gauges. Note however that identification from vibration testing works better with laminate properties rather then ply properties. In the present study we limit the discussion to vibration testing and focus on the two remaining points relative to handling uncertainty.

Numerous improvements in identification methods have strived at providing increasingly accurate estimates of various material properties. A numerical estimate alone for a material property is however of questionable interest. Indeed it is often observed that from a single test the different material properties are not obtained with the same confidence. Typically the shear modulus and the Poisson's ratio are known with significantly less accuracy from a vibration test. Accordingly, estimating the uncertainty with which a property is determined can be as important as estimating its mean or most likely value. A possible natural representation of this uncertainty is through the joint probability distribution function of the properties which provides not only estimates of uncertainty in the properties (variances) but also estimates of the correlation between them (covariances).

Determining the uncertainty in the output values (identified properties) requires however some knowledge of the uncertainty in the input (measurement errors, model errors). Indeed multiple sources of uncertainties are present which have an effect on the identification. First there is the uncertainty in the measured frequencies. Furthermore there are uncertainties on the input parameters of the model (e.g. dimensions and density of the plate). Finally there is uncertainty in the ability of the model to predict the actual experiment. These uncertainties need to be taken into account when identifying the material properties. A possible framework for this is Bayesian. The Bayesian method is a statistical approach to identification handling uncertainty in input parameters and also providing a probability distribution for the output, thus taking care of the two major points mentioned earlier.

Several authors have applied in the past statistical methods to identification, notably Bayesian techniques. Sol was probably among the first to apply a Bayesian approach to identification of the elastic constants of composites [11]. Several other authors have pursued since along this path [12]-[15]. The majority of these articles however used the Bayesian procedure only to obtain a more accurate estimate of the properties. Only [13] provided values of the standard deviations of the identified properties. With respect to uncertainty modeling, all the previous articles considered only errors in frequency measurements, errors which were assumed normally distributed. Such an assumption makes it possible to handle the Bayesian identification in a purely analytical framework with very low computational cost. Handling uncertainty in model input parameters (e.g. plate dimensions) and modeling error requires the use of statistical sampling and simulation thus significantly increasing computational cost. A major challenge lies then in developing techniques that allow the required statistical simulation at reasonable computational cost. To deal with this challenge we propose here a Bayesian procedure based on the use of response surface approximations for the natural frequencies of the plate.

The aim of the present paper is then to identify the probability distribution of the four orthotropic ply elastic constants of a thin composite laminate from natural frequency measurements using a Bayesian approach which can handle all three previously discussed uncertainties: measurement uncertainties on the natural frequencies, uncertainty on the other input parameters involved and modeling uncertainty.

In Section II we describe the vibration problem that serves for material properties identification. Then in Section III the Bayesian identification procedure is described in details, including Bayesian formulation, error model and numerical procedure. In section IV we construct the frequency response surface approximations required for reducing computational cost. Finally we give the Bayesian identification results in Section V. Section VI provides concluding remarks.

\section{Vibration problem}

We seek the four in-plane ply-elastic constants of a thin composite laminate: $E_{1}, E_{2}, G_{12}$ and $v_{12}$. In the present study we use the experimental measurements obtained by Pedersen and Frederiksen [16]. They measured the first ten natural frequencies of a thin glass/epoxy composite laminate with a stacking sequence of $[0,-40,40,90,40,0,90,-$ $40]_{\mathrm{s}}$. The rectangular plate had dimensions (length $a$, width $b$ and thickness $h$ ) given in Table 1. It was attached by two strings which were assumed to be modeled appropriately by free boundary conditions. The measured frequencies are provided in Table 2.

Table 1: Plate properties: length (a), width (b), thickness(h) and density $(\rho)$.

\begin{tabular}{|c|c|c|c|c|}
\hline Parameter & $\mathrm{a}(\mathrm{mm})$ & $\mathrm{b}(\mathrm{mm})$ & $\mathrm{h}(\mathrm{mm})$ & $\rho\left(\mathrm{kg} / \mathrm{m}^{3}\right)$ \\
\hline Value & 209 & 192 & 2.59 & 2120 \\
\hline
\end{tabular}


Table 2 : Experimental frequencies from Pedersen and Frederiksen [16].

\begin{tabular}{|l|c|c|c|c|c|c|c|c|c|c|}
\hline Frequency & $f_{1}$ & $f_{2}$ & $f_{3}$ & $f_{4}$ & $f_{5}$ & $f_{6}$ & $f_{7}$ & $f_{8}$ & $f_{9}$ & $f_{10}$ \\
\hline Value $(\mathrm{Hz})$ & 172.5 & 250.2 & 300.6 & 437.9 & 443.6 & 760.3 & 766.2 & 797.4 & 872.6 & 963.4 \\
\hline
\end{tabular}

In [16] Pedersen and Frederiksen applied a basic least squares approach for identifying the four ply-elastic constants: $\mathrm{E}_{1}, \mathrm{E}_{2}, \mathrm{G}_{12}$ and $v_{12}$. This involved minimizing the following objective function:

$$
J(E)=\sum_{i=1}^{m}\left(\frac{f_{i}^{\text {num }}(E)-f_{i}^{\text {measure }}}{f_{i}^{\text {measure }}}\right)^{2}
$$

where $E=\left\{E_{1}, E_{2}, v_{12}, G_{12}\right\}, f_{i}^{\text {measure }}$ is the $\mathrm{i}^{\text {th }}$ experimental frequency from Table 2 and $f_{i}^{\text {num }}$ is the prediction of a numerical model (a Rayleigh-Ritz method was used in [16]).

\section{Bayesian identification}

\section{A. Fundamental principle}

In the present study we apply the Bayesian approach to the previously described vibration problem to identify the probability distribution of the four ply-elastic constants. It is based on the application of Bayes' rule which gives the probability of an event A, knowing that an event B occurred as shown in Eq. 3. Often $P(A)$ is called the prior probability of $\mathrm{A}, P^{\text {prior }}(A)$, to mark the distinction to the probability of $\mathrm{A}$ knowing $\mathrm{B}$, which is also called probability of A posterior to $\mathrm{B}$.

$$
P(A / B)=\frac{P(B / A) \cdot P(A)}{P(B)}
$$

To apply Bayes' rule to the identification of material properties let us denote $X$ the random variable of the material properties to be identified ( $x$ is an instance of $X$ ). Its probability distribution function (pdf) is denoted $\pi(x)$. Similarly $Y$ is the random variable of the model predictions (i.e., frequencies here) with $\pi(y)$ its pdf. Note that the uncertainty in $Y$ is not necessarily due only to uncertainty in the material properties $X$, but can be due to uncertainty in other parameters (dimensions, loads, etc).

We can now introduce $X / Y=y^{\circ}$, the random variable $X$ given that we observed $Y=y^{\circ} ; \pi\left(x / Y=y^{\circ}\right)$ is its corresponding pdf. Similarly we define $\pi\left(y^{\circ} / X=x\right)$, which is also called likelihood function of $X$ given $y^{\circ}$. We also denote by $\pi^{\text {prior }}(x)$ the pdf of $X$ prior to observing $y^{\circ}$. Bayes' rule extended to continuous probability distributions provides then the distribution of the material properties $x$ given that we observed $Y=y^{\circ}$ by the following equation:

$$
\pi\left(x / Y=y^{o}\right)=\frac{\pi\left(y^{o} / X=x\right) \cdot \pi^{\text {prior }}(x)}{\int \pi\left(y^{o} / X=x\right) \cdot \pi^{\text {prior }}(x) \cdot d x}=\frac{1}{K} \cdot \pi\left(y^{o} / X=x\right) \cdot \pi^{\text {prior }}(x)
$$

where the integral in the denominator represents a normalization constant, denoted as $K$ for simplicity. For more details on the theory of Bayesian analysis refer to [17] and Bayesian identification [18].

Applied to the vibration problem the Bayesian approach can be written as follows.

$$
\pi\left(E / f=f^{\text {measure }}\right)=\frac{1}{K} \pi\left(f=f^{\text {measure }} / E\right) \cdot \pi^{\text {prior }}(E)
$$

where $E=\left\{E_{1}, E_{2}, v_{12}, G_{12}\right\}$ is the four dimensional random variable of the elastic constants, $f=\left\{f_{1} \ldots f_{10}\right\}$ the ten dimensional random variables of the natural frequencies of the plate and $f^{\text {measure }}=\left\{f_{1}^{\text {measure }} \ldots f_{10}^{\text {measure }}\right\}$ the instance of the ten measured natural frequencies.

Equation 4 provides the joint probability distribution of the four elastic constants given the measurements $f^{\text {measure }}$. This pdf is equal to a normalizing constant times the likelihood function of the elastic constants $E$ given the measurements $f^{\text {measure }}$ times the prior distribution of the elastic constants $E$. The prior distribution of $E$ reflects the prior knowledge we have on the elastic constants. This knowledge can come from manufacturer specifications for example. It has a regularization purpose by reducing the likelihood of values of $E$ which are too far off from reasonable values defined by the prior knowledge on the composite studied. In our case we assumed that we only 
have relatively vague prior knowledge by defining a joint normal prior distribution with relatively wide standard deviations as defined in Table 3.

Table 3: Normal uncorrelated prior distribution of the material properties.

\begin{tabular}{|c|c|c|c|c|}
\hline Parameter & $\mathrm{E}_{1}(\mathrm{GPa})$ & $\mathrm{E}_{2}(\mathrm{GPa})$ & $v_{12}$ & $\mathrm{G}_{12}(\mathrm{GPa})$ \\
\hline Mean value & 60 & 21 & 0.28 & 10 \\
\hline Standard deviation & 10 & 5 & 0.05 & 1.5 \\
\hline
\end{tabular}

The other term on the right hand side of Eq. 4 is the likelihood function of the elastic constants given the measurements $f^{\text {measure }}$. This function measures the probability of getting the test results for a given value of the elastic constants $E$, and consequently, it provides an estimate of the likelihood of different $E$ values given the test results. Note that there is randomness in the natural frequencies since for a given value of $E$ we need to calculate the probability of obtaining the measurements. This randomness can have several causes.

A typical cause is measurement error. Previous studies [11]-[15] assumed this to be the only uncertainty and also made the assumption that it is normally distributed. These assumptions allow a purely analytical treatment of the likelihood function, which has the advantage of reduced computational cost. In our study we assume uniformly distributed measurement error. Note that from the information we have available we do not have a way of determining whether uniform uncertainty is more likely on our problem than normal uncertainty or vice-versa. Determining the exact uncertainty structure would require an in depth analysis of the possible sources of uncertainty in the frequency measurements procedure. If we had this uncertainty structure we could directly incorporate it into our Bayesian procedure which uses Monte Carlo simulation for propagating uncertainties. This is unlike analytical Bayesian procedures which could handle only Gaussian measurement uncertainty structures. To illustrate the capability of our procedure to handle other types of uncertainty we chose to model uniform measurement uncertainty.

The numerical treatment, based on Monte Carlo simulation also allows us to consider other types of uncertainties, one of it being modeling error. One potential modeling error is due to the use of thin plate theory, which is more severe for higher modes due to the lower wave length of the higher modes. Other modeling errors, such as discretization errors also increase for higher modes due to more complex mode shapes.

Finally yet another uncertainty in the identification process is due to uncertainty in the dimensions of the plate and its density. We develop an error model for these parameters in the next section and use Monte Carlo simulation to propagate their effect to the natural frequencies and obtain the likelihood function.

\section{B. Error model}

We chose to model measurement and modeling error together as following. We assume that the natural frequencies have a uniformly distributed error:

$$
f_{m}=f_{m}^{\text {response }}+u_{m}
$$

where $f_{m}{ }^{\text {response }}$ is the plate's $m^{\text {th }}$ natural frequency obtained from a thin plate theory model and $u_{m}$ is a uniformly distributed random variable modelling the error.

This error was assumed not to be centered in the experimental frequencies (i.e. the mean of $u_{m}$ is not zero) due to the thick-thin plate modeling error and the discretization error. Indeed even though we used a thin plate (see dimensions in Table 1) transverse shear effects become non-negligible especially for higher modes. The difference remains small however and will change little as the elastic constants change during the updating procedure. Accordingly we evaluated this difference, that was assumed not to vary during the updating, using the mean values of the a priori distribution of the four in-plane properties (see Table 3). For the transverse shear values we considered $G_{13}=G_{12}$ and $G_{23}=0.9 G_{12}$, typical for such a glass/epoxy composite. We found following differences.

Note that thick plate theory could have been used directly in the numerical model. However we chose thin plate theory for the numerical model because we need to construct response surface approximations of the frequencies in order to reduce computational cost. As will be shown in Section 4 the thin plate theory natural frequencies can be expressed with great accuracy in a relatively simple polynomial form. The more complex governing equations of the thick plate theory would have required more complex response surface approximations. Accordingly we chose to use thin plate theory and model the difference to thick plate theory through the systematic error model described. 
Table 4 : Difference between the frequencies obtained with thin plate theory and thick plate theory for the mean values of the a priori material properties (see Table 3 ) and $\mathrm{G}_{13}=\mathrm{G}_{12}$ and $\mathrm{G}_{23}=0.9 \mathrm{G}_{12}$.

\begin{tabular}{|l|c|c|c|c|c|c|c|c|c|c|}
\hline Frequency & $f_{1}$ & $f_{2}$ & $f_{3}$ & $f_{4}$ & $f_{5}$ & $f_{6}$ & $f_{7}$ & $f_{8}$ & $f_{9}$ & $f_{10}$ \\
\hline Difference & $0.31 \%$ & $0.03 \%$ & $0.04 \%$ & $0.29 \%$ & $0.29 \%$ & $0.10 \%$ & $0.07 \%$ & $0.42 \%$ & $0.32 \%$ & $0.23 \%$ \\
\hline
\end{tabular}

Since during the Bayesian updating procedure we use a thin plate model the difference in Table 4 defines the center of the uniformly distributed error that we assume (see Eq. 5). To fully define the error model we now need to define the bounds of the uniform distribution. We consider that the bounds of the distribution increase with the mode number, which can be explained as follows:

i. due to noise it is harder to numerically extract the higher frequencies

ii. the higher modes, due to their higher frequencies and more complex mode shapes may start involving effects which were considered negligible for the fundamental frequency (surrounding air effects, damping, more complex material behavior)

Accordingly we considered that the upper/lower bound is $+/-0.5 \%$ of the measured experimental frequency for the fundamental mode $(\mathrm{m}=1)$ and $+/-2 \%$ for the highest mode measured $(\mathrm{m}=10)$. The bounds vary linearly with $m$ in-between.

Apart from the error model just defined, which accounts for modeling and measurement uncertainty, we also modeled the uncertainty in the input parameters of the thin plate model. The thin plate model involves apart from the four material properties four other parameters: the plate length, width and thickness $(a, b$ and $h)$ and the plate density $\rho$. These parameters are measured beforehand and are known only with a certain confidence. We assumed following uncertainties on these parameters.

Table 5: Assumed uncertainties in the plate length, width, thickness and density $(a, b, h$ and $\rho)$. Normal distributions are used.

\begin{tabular}{|l|c|c|c|c|}
\hline Parameter & $a(\mathrm{~mm})$ & $b(\mathrm{~mm})$ & $h(\mathrm{~mm})$ & $\rho\left(\mathrm{kg} / \mathrm{m}^{3}\right)$ \\
\hline Mean value & 209 & 192 & 2.59 & 2120 \\
\hline Standard deviation & 0.25 & 0.25 & 0.01 & 10.6 \\
\hline
\end{tabular}

\section{Bayesian numerical procedure}

The Bayesian identification involves evaluating the posterior distribution defined in Eq. 4. The prior distribution in the right hand side is an analytical expression (normal distribution characterized in Table 3) so the major challenge is in constructing the likelihood function. We chose to construct this function point by point: we evaluate it at a grid in the four-dimensional space of the material properties $E=\left\{E_{1}, E_{2}, v_{12}, G_{12}\right\}$. We chose here an $8^{4}$ grid, which is determined by computational cost considerations. A convergence study is given in Section 5 .

At each of the grid points $E$ is fixed and we need to evaluate the probability distribution function (pdf) of the natural frequencies, $\pi\left(f / E^{f i x e d}\right)$, at the point $f=f^{\text {measure }}$. The pdf of the natural frequencies is determined by propagating through Monte Carlo simulation the uncertainties in the input parameters and the error model defined in the previous subsection. This is done in two steps for each $E$ point on the grid:

a. Propagate the normal uncertainties on the input parameters (see Table 5) to the natural frequencies using Monte Carlo simulation. We denote $f^{\text {input }} M C$ the random variable of the frequencies due only to the uncertainty in input parameters but not to the measurement uncertainty.

b. Calculate the point of the likelihood function using Eq. 6 which accounts for the measurement and modeling uncertainty $u$ (see Eq. 5).

$$
\pi\left(f=f^{\text {measure }} / E^{\text {fixed }}\right)=\int_{f^{\text {measwer }}-u^{\text {lb }}}^{f_{\text {massure }}^{\text {ubb }}} \pi\left(f^{\text {input_MC }} / E^{\text {fixed }}\right) d f^{\text {input_MC }}
$$

The use of Eq. 6 is possible because the combined measurement and modeling error is uniformly distributed (uniform distribution within the bounds $\left[u^{l b}, u^{u b}\right]$ ). Equation 6 is equivalent to saying that the likelihood of measuring the frequencies $f^{\text {measure }}$ is equal to the probability that the simulated frequencies $f^{\text {jnput } \_M C}$ fall inside the measurement uncertainty bounds $\left[f^{\text {neasure }}-u^{l b}, f^{\text {neasure }}+u^{u b}\right]$. The integral in Eq. 6 is evaluated by counting the number of frequencies within the bounds $\left[f^{\text {measure }}-u^{l b}, f^{\text {measure }}+u^{u b}\right]$ out of the total number of simulated frequencies $f^{\text {input }-M C}$. We used 50,000 Monte Carlo simulations.

In a more general case where the measurement uncertainty is not uniformly distributed such as to allow the use of Eq. 6 following procedure can be applied instead. For each fixed $E$ point on the grid simulate values for the 
uncertain input parameters $a, b, h$ and $\rho$, propagate these to the natural frequencies and add a sampled value for the modeling and measuring uncertainty $u$. This will lead to a sample of simulated frequencies. We need to fit a distribution to these frequencies and evaluate it at $f=f^{\text {measure }}$, which would lead to the likelihood value we seek $\pi(f$ $\left.=f^{\text {neasure }} / E^{f \text { ixed }}\right)$. To fit the sampled frequencies we can construct the empirical histogram and fit it using a kernel method for example.

In all the cases, since the Bayesian updating procedure used involves Monte Carlo simulation we are confronted with significant computational cost. For each of the $8^{4}$ grid points 50,000 Monte Carlo simulations of the first 10 natural frequencies are carried out. This means that in total we have about 2 billion frequency evaluations. In such a context it is obvious that a numerical model (such as finite elements) is by far too expensive for calculating the natural frequencies. To reduce the cost we chose to construct response surface approximations of the frequencies. Response surface approximations are surrogates of the actual response obtained from a small number of sampling points. The surrogate expression replaces the more expensive numerical model over a given construction domain. Typical surrogates are polynomial response surfaces, kriging and neural networks. For additional details on the response surface methodology refer to [19].

The response surface method allows to reduce the cost of a frequency evaluation from about $4 \mathrm{~s}$ using the finite element model to about $0.3 \mathrm{~ms}$ with the surrogate model (on an Intel Core2 Duo 2GHz machine). This brings the computational cost of the Bayesian procedure down to tractable levels.

\section{Natural frequencies response surface approximations}

\section{A. Nondimensionalization for number of variables reduction}

We need to construct response surface approximations (RSA) of the first ten natural frequencies of the plate which can account for variations in the four elastic constants $E_{1}, E_{2}, v_{12}, G_{12}$ as well as variations in the uncertain parameters: plate dimensions $a, b$ and $h$ and plate density $\rho$. We could construct the RSAs as a function of these seven parameters directly. However, in order to improve accuracy and reduce computational cost we chose to construct the RSA as a function of the nondimensional parameters characterizing the vibration problem. To find these nondimensional parameters we nondimensionalize the governing equation and the boundary conditions of the problem.

The governing equation for the symmetric laminate we consider can be written:

$$
D_{11} \frac{\partial^{4} w}{\partial x^{4}}+4 D_{16} \frac{\partial^{4} w}{\partial x^{3} \partial y}+2\left(D_{12}+2 D_{66}\right) \frac{\partial^{4} w}{\partial x^{2} \partial y^{2}}+4 D_{26} \frac{\partial^{4} w}{\partial x \partial y^{3}}+D_{22} \frac{\partial^{4} w}{\partial y^{4}}+\rho h \frac{\partial^{2} w}{\partial t^{2}}=0
$$

where $w$ is the out of plane displacement, $\rho$ the mass density, $h$ the plate thickness and $D_{i j}$ the rigidities of the plate. The bending rigidities $D_{i j}$ are a function of the individual in plane properties of a ply $\left(E_{1}, E_{2}, v_{12}\right.$ and $\left.G_{12}\right)$, the thickness $h$ and the composite layup orientation. For the detailed expressions of the rigidities of a composite laminate refer to [20].

The free boundary conditions are modeled as following:

On edge $\mathrm{x}=0$ and $\mathrm{x}=\mathrm{a}($ denoted $\mathrm{x}=0 ; \mathrm{a})$ :

$$
\begin{aligned}
& M_{x}=0 \quad \Leftrightarrow \quad-\left.D_{11} \frac{\partial^{2} w}{\partial x^{2}}\right|_{x=0 ; a}-\left.D_{12} \frac{\partial^{2} w}{\partial y^{2}}\right|_{x=0 ; a}=0 \\
& Q_{x}+\frac{\partial M_{x y}}{\partial y}=0 \quad \Leftrightarrow \quad-\left.D_{11} \frac{\partial^{3} w}{\partial x^{3}}\right|_{x=0 ; a}-\left.\left(D_{12}+4 D_{66}\right) \frac{\partial^{3} w}{\partial x \partial y^{2}}\right|_{x=0 ; a}=0
\end{aligned}
$$

On edge $\mathrm{y}=0$ and $\mathrm{y}=\mathrm{b}($ denoted $\mathrm{y}=0 ; \mathrm{b})$ :

$$
\begin{aligned}
& M_{y}=0 \quad \Leftrightarrow \quad-\left.D_{12} \frac{\partial^{2} w}{\partial x^{2}}\right|_{y=0 ; b}-\left.D_{22} \frac{\partial^{2} w}{\partial y^{2}}\right|_{y=0 ; b}=0 \\
& Q_{y}+\frac{\partial M_{x y}}{\partial x}=0 \quad \Leftrightarrow \quad-\left.D_{22} \frac{\partial^{3} w}{\partial y^{3}}\right|_{y=0 ; b}-\left.\left(D_{12}+4 D_{66}\right) \frac{\partial^{3} w}{\partial x^{2} \partial y}\right|_{y=0 ; b}=0
\end{aligned}
$$

The whole vibration problem (governing equation and boundary conditions) thus involves 13 variables to which we add the variable of the natural frequencies $f_{m}$ that we seek, so a total of 14 variables for the problem of determining the plate's natural frequency (see Table 6). 
Table 6: Variables involved in the vibration problem and their units

\begin{tabular}{|l|c|c|c|c|c|c|c|c|c|c|c|c|l|l|}
\hline Variable & $f_{m}$ & $w$ & $x$ & $y$ & $a$ & $b$ & $t$ & $\rho h$ & $D_{11}$ & $D_{12}$ & $D_{22}$ & $D_{66}$ & $D_{16}$ & $D_{26}$ \\
\hline Unit & $\frac{1}{s}$ & $m$ & $m$ & $m$ & $m$ & $m$ & $s$ & $\frac{\mathrm{kg}}{\mathrm{m}^{2}}$ & $\frac{\mathrm{kg} \cdot \mathrm{m}^{2}}{\mathrm{~s}^{2}}$ & $\frac{\mathrm{kg} \cdot \mathrm{m}^{2}}{\mathrm{~s}^{2}}$ & $\frac{\mathrm{kg} \cdot \mathrm{m}^{2}}{\mathrm{~s}^{2}}$ & $\frac{\mathrm{kg} \cdot \mathrm{m}^{2}}{\mathrm{~s}^{2}}$ & $\frac{\mathrm{kg} \cdot \mathrm{m}^{2}}{\mathrm{~s}^{2}}$ & $\frac{\mathrm{kg} \cdot \mathrm{m}^{2}}{\mathrm{~s}^{2}}$ \\
\hline
\end{tabular}

These 14 variables involve 3 independent dimension groups $(m, k g, s)$. According to the Vaschy-Buckingham theorem we know that we can have a minimum of $14-3=11$ nondimensional groups.

Defining $\tau=\sqrt{\frac{\rho h a^{4}}{D_{11}}}$, which can be viewed as a time constant, the 11 nondimensional groups can be expressed as:

$$
\begin{aligned}
& \Omega=\frac{w}{h} \quad \theta=\frac{t}{\tau} \quad \xi=\frac{x}{a} \quad \eta=\frac{y}{b} \quad \gamma=\frac{a}{b} \\
& \Psi_{m}=\tau f_{m} \quad \Delta_{12}=\frac{D_{12}}{D_{11}} \quad \Delta_{22}=\frac{D_{22}}{D_{11}} \quad \Delta_{66}=\frac{D_{66}}{D_{11}} \quad \Delta_{16}=\frac{D_{16}}{D_{11}} \quad \Delta_{26}=\frac{D_{26}}{D_{11}}
\end{aligned}
$$

In terms of these nondimensional variables the vibration problem can be written as follows:

Governing equation:

$$
\frac{\partial^{4} \Omega}{\partial \xi^{4}}+4 \Delta_{16} \gamma \frac{\partial^{4} \Omega}{\partial \xi^{3} \partial \eta}+2\left(\Delta_{12}+2 \Delta_{66}\right) \gamma^{2} \frac{\partial^{4} \Omega}{\partial \xi^{2} \partial \eta^{2}}+4 \Delta_{26} \gamma^{3} \frac{\partial^{4} \Omega}{\partial \xi \partial \eta^{3}}+\Delta_{22} \gamma^{4} \frac{\partial^{4} \Omega}{\partial \eta^{4}}+\frac{\partial^{2} \Omega}{\partial \theta^{2}}=0
$$

Boundary conditions:

On edge $\xi=0$ and $\xi=1($ denoted $\xi=0 ; 1)$ :

$$
\begin{aligned}
& -\left.\frac{\partial^{2} \Omega}{\partial \xi^{2}}\right|_{\xi=0 ; 1}-\left.\Delta_{12} \gamma^{2} \frac{\partial^{2} \Omega}{\partial \eta^{2}}\right|_{\xi=0 ; 1}=0 \\
& -\left.\frac{\partial^{3} \Omega}{\partial \xi^{3}}\right|_{\xi=0 ; 1}-\left.\left(\Delta_{12}+4 \Delta_{66}\right) \gamma^{2} \frac{\partial^{3} \Omega}{\partial \xi \partial \eta^{2}}\right|_{\xi=0 ; 1}=0
\end{aligned}
$$

On edge $\eta=0$ and $\eta=1$ (denoted $\eta=0 ; 1)$ :

$$
\begin{aligned}
& -\left.\Delta_{12} \frac{\partial^{2} \Omega}{\partial \xi^{2}}\right|_{\eta=0 ; 1}-\left.\Delta_{22} \gamma^{2} \frac{\partial^{2} \Omega}{\partial \eta^{2}}\right|_{\eta=0 ; 1}=0 \\
& -\left.\Delta_{22} \gamma^{3} \frac{\partial^{3} \Omega}{\partial \eta^{3}}\right|_{\eta=0 ; 1}-\left.\left(\Delta_{12}+4 \Delta_{66}\right) \gamma \frac{\partial^{3} \Omega}{\partial \xi^{2} \partial \eta}\right|_{\eta=0 ; 1}=0
\end{aligned}
$$

We are interested in our problem in expressing the nondimensional natural frequency $\Psi_{m}$. We are not interested in the vibration mode shapes and we know that the natural frequency will not depend on the nondimensional out-ofplane displacement $\Omega$, nor on the nondimensional time $\theta$, nor on the nondimensional coordinates $\xi$ and $\eta$. This means that the nondimensional natural frequency $\Psi_{m}$ will be a function of only six nondimensional parameters $\Delta_{12}$, $\Delta_{22}, \Delta_{66}, \Delta_{16}, \Delta_{26}$ and $\gamma$ :

$$
\Psi_{m}=f\left(\Delta_{12}, \Delta_{22}, \Delta_{66}, \Delta_{16}, \Delta_{26}, \gamma\right)
$$

Approximate analytical solutions for the vibration problem [21] hinted that a third degree polynomial approximation of $\Psi^{2}$ as a function of $\Delta_{12}, \Delta_{22}, \Delta_{66}, \Delta_{16}, \Delta_{26}$ and $\gamma^{2}$ could lead to reasonable results. Accordingly we will seek to construct a response surface approximation under the following form:

$$
\Psi_{m}{ }^{2}=\operatorname{PRS}\left(\Delta_{12}, \Delta_{22}, \Delta_{66}, \Delta_{16}, \Delta_{26}, \gamma^{2}\right)
$$




\section{B. Response surface construction}

For each of the ten modes we construct a third degree polynomial response surface (PRS) approximation of the squared nondimensional natural frequency $\Psi^{2}$. The PRS is fitted to a finite element (FE) model of a plate in Abaqus ${ }^{\circledR}$ commercial FE software using 400 thin plate S8R5 elements.

In order to construct the response surface approximations we need to sample points in the six-dimensional space of the nondimensional parameters at which we will evaluate the FE response. If we sample in the nondimensional variables directly it is however difficult to deduce values for the dimensional variables that need to be provided to the FE model. Accordingly we chose the following procedure to sample the points in the nondimensional space:

i. Sample 5000 points in the eight dimensional-variables space $\left\{E_{1}, E_{2}, v_{12}, G_{12}, a, b, h, \rho\right\}$ using the uncertainties defined in Table 5 for the plate length, width, thickness and density and using uniform uncertainties for $E_{1}, E_{2}, v_{12}, G_{12}$ (defined as uniformly distributed between bounds at +/- two standard deviations of the prior distribution of $E$ ).

ii. Out of the 5000 points extract 200 according to the max-min criterion in the nondimensional variable space using the Matlab Surrogates Toolbox [22]. The max-min criterion aims at maximizing the minimum distance between any two points. This steps ensures that the points are well distributed (space-filling) in the nondimensional variables space in which the response surface approximations will be constructed.

Figure 1 illustrates this procedure in a case where we would have only two non-dimensional variables: $\Delta_{12}$ and $\gamma$. The blue crosses are representative of the 5000 points sampled in step i. The red circles are representative of the 200 points selected in step two according to the max-min criterion. Note that due to the fact that we need to stop the max-min criterion after a certain number of iterations (to keep computational cost reasonable) we are not guaranteed to obtain the absolute maximum. However even if not the absolute maximum this procedure ensures that the points are reasonably well distributed in the nondimensional space of interest for the response surface construction.

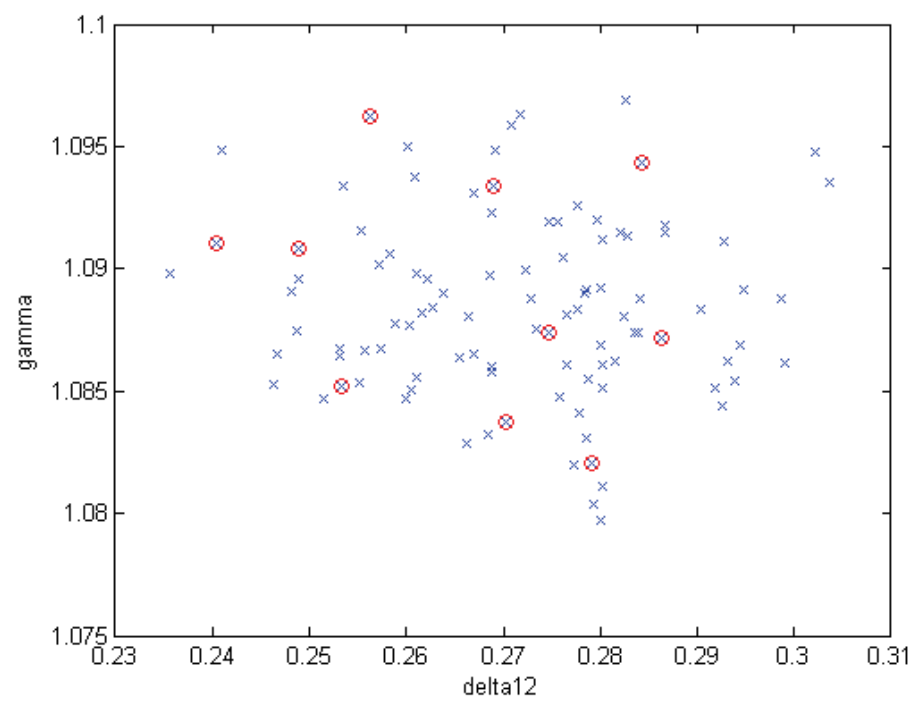

Figure 1: Illustration of the procedure for sampling points in the nondimensional space for the RSA construction.

Having sampled the 200 points in the nondimensional space and knowing the corresponding dimensional parameters we can calculate the FE response at these points and fit the cubic polynomial response surfaces (PRS) for each of the first ten natural frequencies.

Initial results showed that the terms $\Delta_{16}, \Delta_{26}$ seem to have negligible effect on the frequencies. This was confirmed when we fitted a third degree PRS only in terms of $\Delta_{12}, \Delta_{22}, \Delta_{66}$, and $\gamma^{2}$. Even with only four variables we obtained very good quality of the fits for all ten PRS, almost same as when using all six variables. Out of the ten, the worst fit was for the $9^{\text {th }}$ frequency which had following error measures: $\mathrm{R}^{2}=0.99998$ and the RMS error was $0.01 \%$ of the response mean value and $0.42 \%$ of the response standard deviation. This constitutes an excellent fit and all ten RSAs were considered acceptable for the use in the Bayesian procedure. 
Note that a small additional step was required to obtain a good quality of the fit for all ten frequency RSAs. Indeed, initially the RSAs for the frequencies number four to seven were very poor. Typical values for these frequencies are as in Table 2. We can see that frequencies four and five are relatively close as are six and seven. This is because the corresponding modes are symmetric relatively to the $\mathrm{x}$ and $\mathrm{y}$ axis and the aspect ratio of the plate is close to one. For each of the 200 sampling points the dimension parameters vary slightly and for some of these points the two symmetric modes switch, meaning that the x-symmetric mode becomes lower in frequency than the y-symmetric mode for some points and not for others. This issue of switching modes was resolved by modeling only half of the plate and using symmetry boundary conditions for constructing the RSA for frequencies four to seven. Xsymmetry boundary conditions allowed to construct the RSA of the same mode consistently and similarly with ysymmetry.

\section{Bayesian identification results}

To serve as a comparison point for the Bayesian results we provide first the least squares identified values. The least squares results are given in Table 7 and were obtained using the least square formulation described in section 2 and the frequency RSAs obtained in Section 4.

Table 7: Least squares identified properties.

\begin{tabular}{|c|c|c|c|c|}
\hline Parameter & $\mathrm{E}_{1}(\mathrm{GPa})$ & $\mathrm{E}_{2}(\mathrm{GPa})$ & $v_{12}$ & $\mathrm{G}_{12}(\mathrm{GPa})$ \\
\hline Value & 60.9 & 22.7 & 0.217 & 9.6 \\
\hline
\end{tabular}

Applying the Bayesian procedure described in section 3 we obtained following results. The identified posterior distribution is represented graphically in Figure 2 by fixing two of the material properties at the mean values of the posterior distribution and plotting the posterior distribution as a function of the remaining two properties.
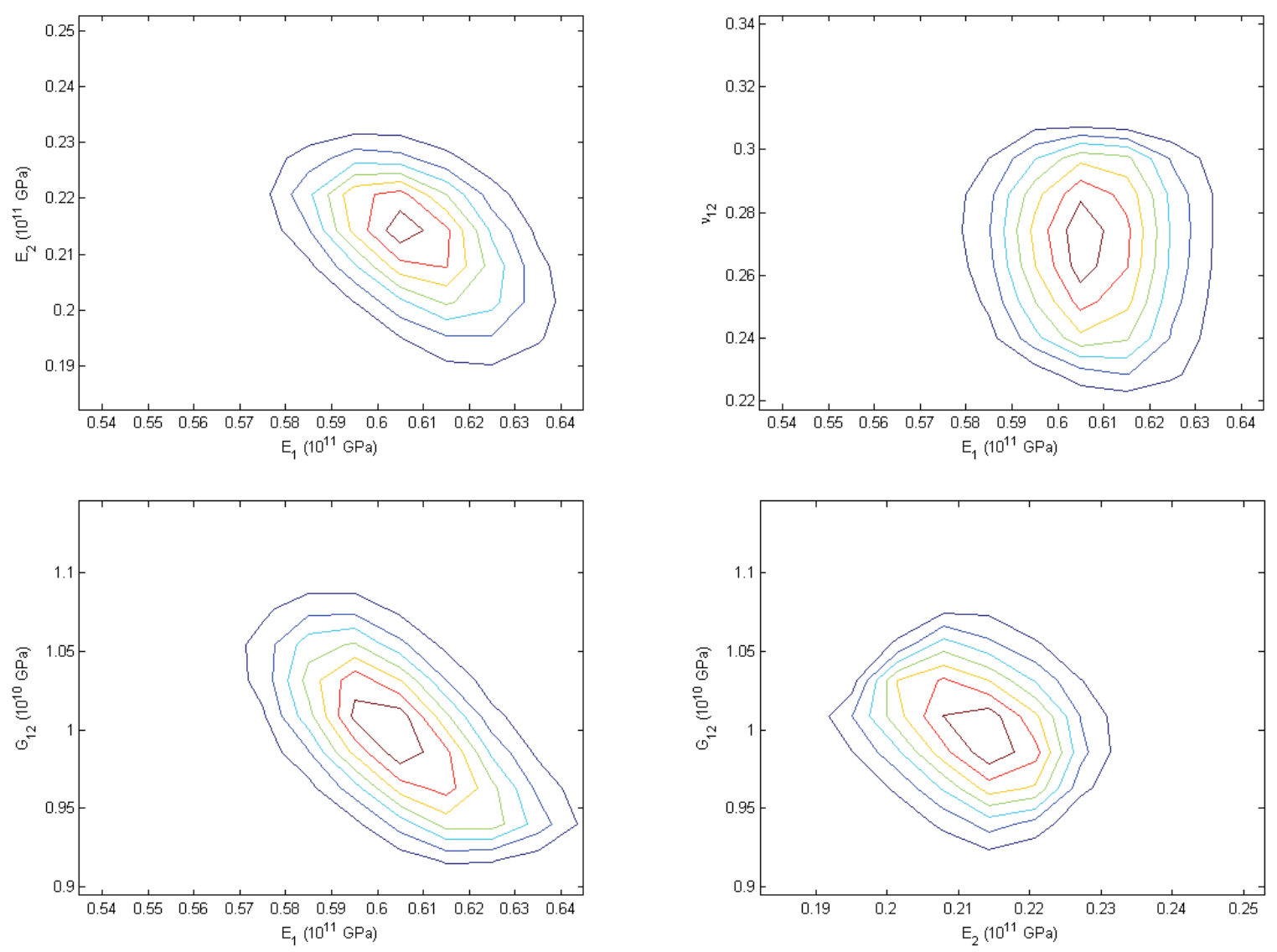

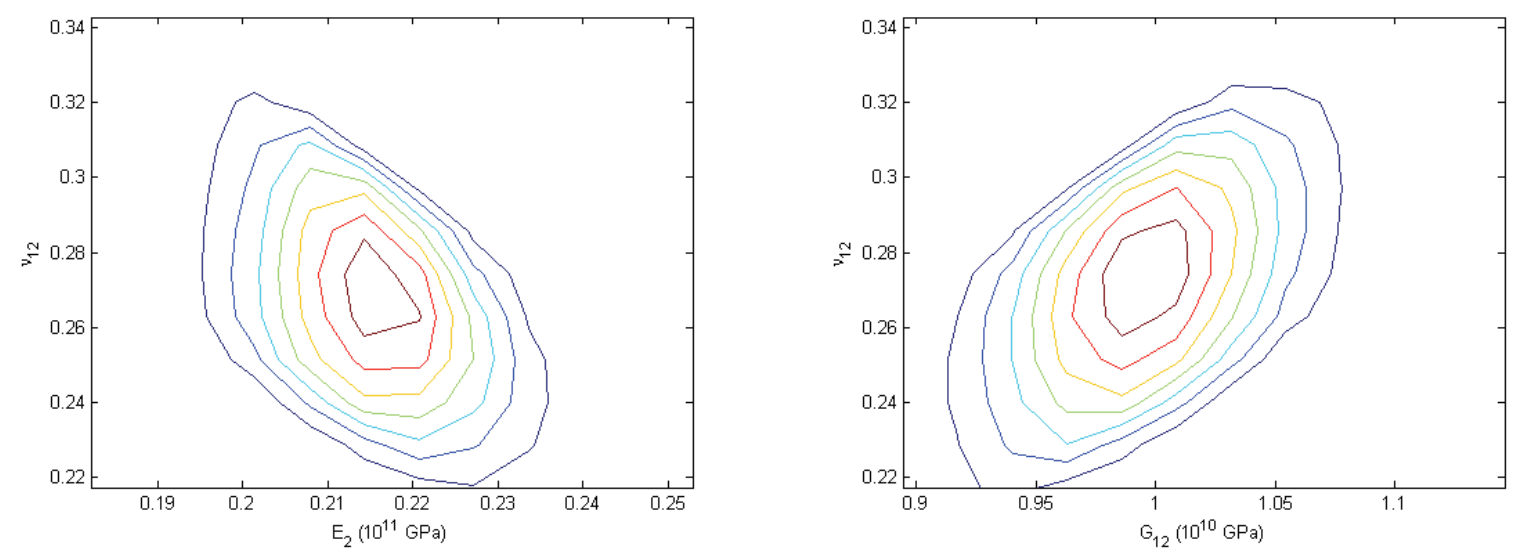

Figure 2: Identified posterior joint distribution plots. Since the joint probability distribution is four-dimensional it is plotted in $2 \mathrm{D}$ by fixing the two variables not plotted at their mean values. Accordingly the plots are six cuts through the distribution mean value point.

We can characterize the identified posterior distribution by the mean values and the variance-covariance matrix as shown in Tables 8 and 9. Since interpreting the variance-covariance matrix is not very easy we also provided the coefficient of variation and the correlation coefficients in the same tables, which facilitate the interpretation of the results. Note that because the variance-covariance and the correlation matrices are symmetric, providing only half of the matrix is sufficient. This is used in Table 9 where the variance-covariance is given in the lower triangular matrix (including diagonal terms) while the correlation coefficient is given in the upper triangular matrix.

Table 8: Mean values and coefficient of variation of the identified posterior distribution.

\begin{tabular}{|c|c|c|c|c|}
\hline Parameter & $\mathrm{E}_{1}(\mathrm{GPa})$ & $\mathrm{E}_{2}(\mathrm{GPa})$ & $v_{12}$ & $\mathrm{G}_{12}(\mathrm{GPa})$ \\
\hline Mean value & 60.8 & 21.3 & 0.27 & 9.87 \\
\hline COV $(\%)$ & 3.05 & 5.46 & 12.2 & 5.96 \\
\hline
\end{tabular}

Table 9: Variance-covariance (lower triangular matrix) and correlation coefficient (upper triangular matrix) of the identified posterior distribution. Variance-covariance coefficients are provided with the units shown in the table while correlation coefficients are nondimensional.

\begin{tabular}{|c|c|r|r|r|}
\hline & \multicolumn{1}{|c|}{$\mathrm{E}_{1}(\mathrm{~Pa})$} & \multicolumn{1}{c|}{$\mathrm{E}_{2}(\mathrm{~Pa})$} & \multicolumn{1}{c|}{$v_{12}$} & \multicolumn{1}{c|}{$\mathrm{G}_{12}(\mathrm{~Pa})$} \\
\hline $\mathrm{E}_{1}(\mathrm{~Pa})$ & $3.45 \mathrm{E}+18$ & -0.141 & -0.378 & -0.626 \\
\hline $\mathrm{E}_{2}(\mathrm{~Pa})$ & $-3.05 \mathrm{E}+17$ & $1.36 \mathrm{E}+18$ & -0.593 & -0.358 \\
\hline$v_{12}$ & $-2.31 \mathrm{E}+07$ & $-2.28 \mathrm{E}+07$ & $1.10 \mathrm{E}-03$ & 0.768 \\
\hline $\mathrm{G}_{12}(\mathrm{~Pa})$ & $-6.85 \mathrm{E}+17$ & $-2.46 \mathrm{E}+17$ & $1.49 \mathrm{E}+07$ & $3.47 \mathrm{E}+17$ \\
\hline
\end{tabular}

A convergence study was carried out in order to estimate the error in the parameters characterizing the identified probability distribution function. Mean values and variance-covariance matrix are indeed dependent on the pdf domain used to calculate them. Since it is computationally impossible to calculate the pdf over the entire domain from $-\infty$ to $\infty$ we need to truncate the pdf when calculating mean and variance-covariance martix. Initially we started with and $8^{4}$ grid which was increased by two points at each step of the convergence study. Note that the step size remained the same, that is we do not refine the domain but enlarge it during the convergence study. The final domain used a $16^{4}$ grid with the domain bounds being those of the plot windows in Figure 2.

The convergence results are illustrated in Figure 3. As far as mean values convergence (Figure 3A) we can say that the results are relatively stable and the estimates are very close to convergence. The highest error seems to be in the shear modulus, which has an estimated truncation error of less than $0.4 \%$.

The variance-covariance matrix on the other hand seems less converged. Regarding standard deviations the highest error seems to be again relative to $G_{I 2}$ which has an estimated truncation error in the standard deviation of less than $8 \%$. The truncation error in the correlation coefficients is estimated to be less than $5 \%$ except for the 
correlation coefficient between $E_{1}$ and $E_{2}$ which seems poorly converged. Note however that these two parameters have the lowest correlation among all so the accuracy on this coefficient is less critical.

The truncation errors could have been further reduced by continuing to enlarge the calculation domain. Since we are in a four dimensional space however, adding points quickly becomes computationally prohibitive. In our case moving from a 16 to an 18 points grid would have required an additional week of calculations on an Intel Core2 Duo $2 \mathrm{GHz}$ machine in spite of the use of response surface approximations. Accordingly we decided to stop at a $16^{4}$ grid. It is however important to note that only the variance co-variance matrix is estimated with somewhat larger errors. The mean values are estimated very accurately. Considering that the variance-covariance matrix is rarely provided at all in identification studies we consider important to estimate it even if only with about $10 \%$ accuracy.

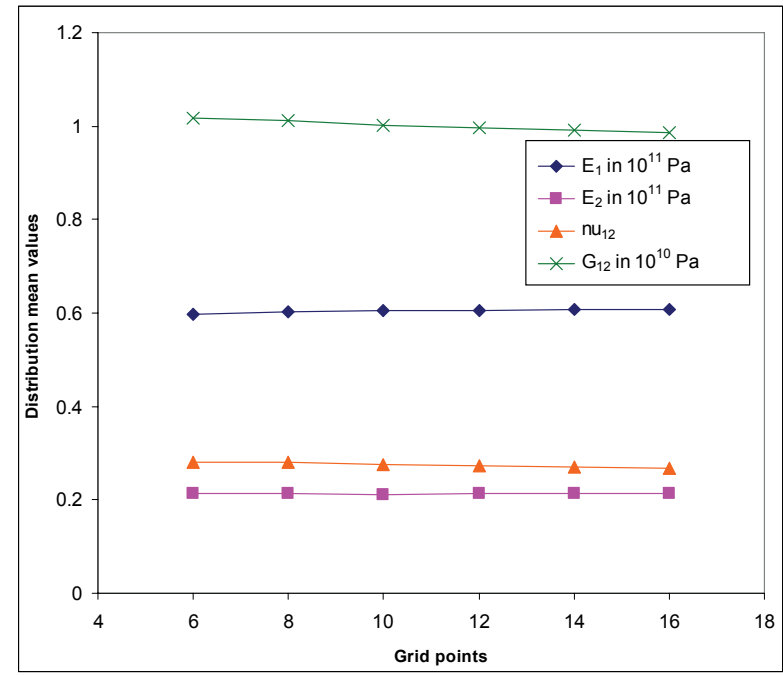

A

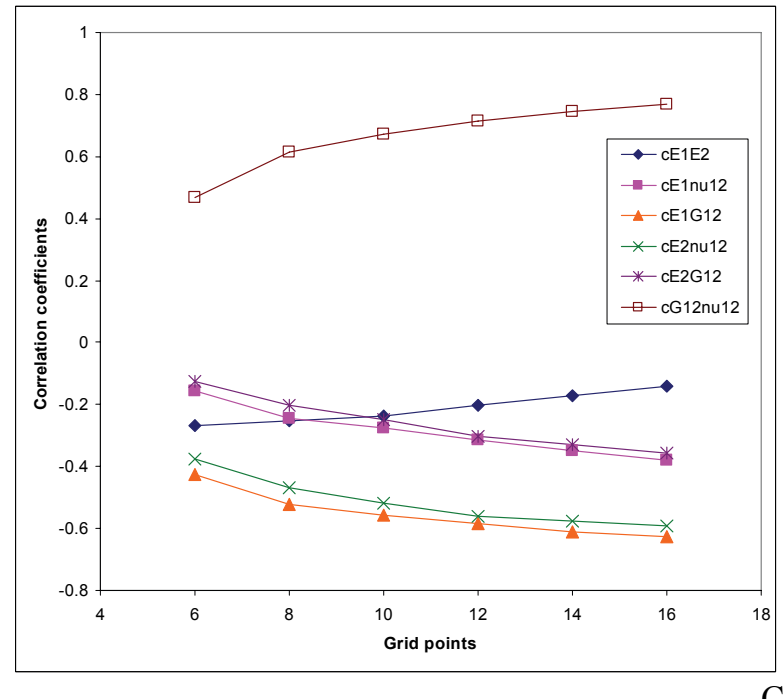

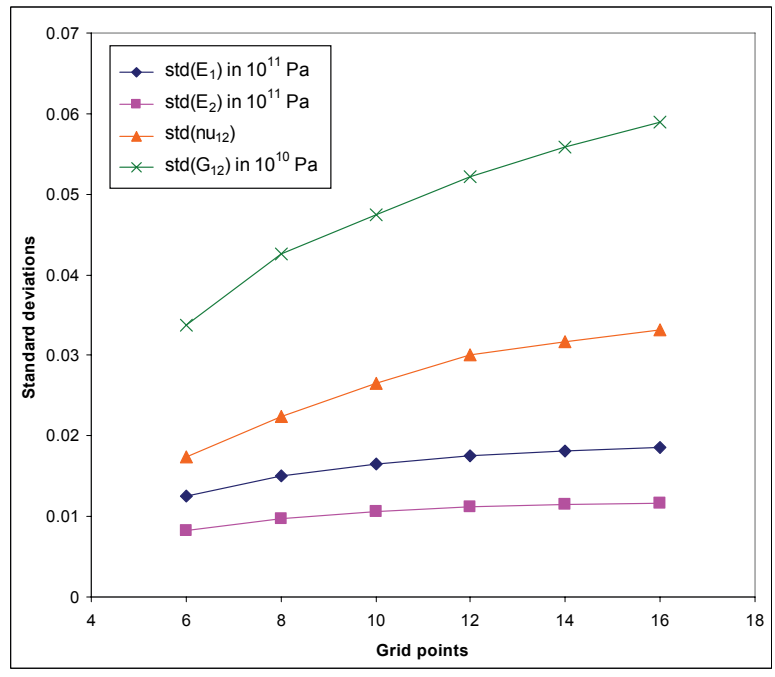

$\mathrm{B}$

Figure 3: Convergence of the identified distribution's mean value (A), standard deviation (B) and correlation coefficient (C).

Comparing Tables 7 and 8 we can note that the least square identified values and the mean values of the posterior distribution are relatively far apart for some properties. However, considering the uncertainties characterizing the posterior distribution and since the least squares values also have an uncertainty at least as high as the Bayesian values, typically higher [23], the results of the two methods seem plausible.

The results of Table 8 show that the four material properties are identified with different confidence from the vibration test we used. The longitudinal Young's modulus is identified most accurately while the Poisson's ratio is identified with a high uncertainty. This trend has been often observed in the composites community, since repeated tests on a same specimen typically lead to much higher dispersion on some properties than on others (Poisson's ratio and shear modulus are typically known more poorly). However it is rarer that the difference in uncertainty is 
quantified and the Bayesian identification approach provides a natural tool for this purpose. Note that the uncertainties depend on the experiment and a different lay-up or another experimental technique would lead to different uncertainties. Note also that the identified pdf is not a representation of the variability of the material properties, it just provides the uncertainty with which they are identified from this particular experiment.

Table 9 shows that there is also non-negligible correlation between the different properties. This is an important result and we could not find any previous study giving the correlation matrix of the orthotropic constants identified. Ignoring the correlation would lead to significantly overestimating the uncertainty in the identified properties. On a final note, the results of Table 8 and 9 can provide a realistic model of experimental uncertainty which can be used in probabilistic studies instead of simple uncorrelated uncertainty models.

\section{Conclusions}

A Bayesian approach for identifying the probability distribution of the orthotropic constants of a composite from natural frequency measurements of a plate was presented. The proposed approach can handle measurement as well as modeling uncertainty through the use of Monte Carlo simulation. Due to the high computational cost of the simulation, response surface approximations of the frequencies were required. Polynomial response surfaces of the natural frequencies as a function of nondimensional parameters were used, which brought down the computational cost by a factor of about 1,000 making the procedure computationally tractable. However, in spite of the substantial cost reduction, the procedure remains at the limit of reasonable computation time. The identified Bayesian posterior distribution was found to have different uncertainties in the different orthotropic constants as well as non-negligible correlation between them. These uncertainties and correlations were quantified and allow the construction of an experimental uncertainty model for the particular experiment used.

\section{References}

[1] Grediac, M. and Vautrin, A., "A new method for determination of bending rigidities of thin anisotropic plates", ASME J. Appl. Mech., Vol 57, pp. 964-968, 1990.

[2] Kernevez, J.P., Knopf-Lenoir Vayssade, C., Touzot, G. et al., "An identification method applied to an orthotropic plate bending experiment", International Journal for Numerical Methods in Engineering, Vol. 12, No. 1, 1978, pp. 129-139.

[3] Arafeh, M.H., Knopf-Lenoir Vayssade, C., and Rouger, F., "Conception optimale d'essais de flexion de plaques orthotropes et identification", Comptes Rendus De l'Académie Des Sciences, Vol. 321, Série II b, 1995, pp. 351354.

[4] Le Magorou, L., Bos, F., and Rouger, F., "Identification of constitutive laws for wood-based panels by means of an inverse method", Composites Science and Technology, Vol. 62, No. 4, 2002, pp. 591-596.

[5] Vautrin, A., "Mechanical identification of composite materials and structures", Proc. of the $2^{\text {nd }}$ AsianAustralasian Conference on Composite Materials, Kyongju, Korea, pp.1305-1323, 2000.

[6] De Wilde, W.P., Narmon, B., Sol, H. and Roovers, M., "Determination of the material constants of an anisotropic lamina by free vibration analysis", Proceedings of the $2^{\text {nd }}$ International Modal Analysis Conference, Orlando, FL, pp. 44-49, 1984.

[7] De Wilde, W.P., Sol, H. and Van Overmeire, M., "Coupling of Lagrange interpolation, modal analysis and sensitivity analysis in the determination of anisotropic plate rigidities", Proceedings of the $4^{\text {th }}$ International Modal Analysis Conference, Los Angeles, California, pp. 1058-1063, 1986.

[8] Deobald, L.R. and Gibson, R.F, "Determination of elastic constants of orthotropic plates by a modal analysis/ Rayleigh-Ritz technique", Journal of Sound and Vibration, Vol. 124(2), pp. 269-283, 1988.

[9] Grediac, M. and Paris, P.A.., "Direct identification of elastic constants of anisotropic plates by modal analysis: theoretical and numerical aspects", Journal of Sound and Vibration, Vol. 195(3), pp. 401-415, 1996.

[10] Grediac, M. Fournier, N., Paris, P.A.. and Surrel, Y., "Direct identification of elastic constants of anisotropic plates by modal analysis: experiments and results", Journal of Sound and Vibration, Vol. 210, pp. 645-659, 1998.

[11] Sol, H., "Identification of anisotropic plate rigidities", PhD dissertation, Vrije Universiteit Brussel, 1986.

[12] Papazoglou, V.J., Tsouvalis, N.G., Lazaridis, A.G., "A non destructive evaluation of the material properties of a composite laminated plate", Applied Composite Materials, Vol. 3, pp. 321-334, 1996.

[13] Lai, T.C. and Ip, K.H., "Parameter estimation of orthotropic plates by Bayesian sensitivity analysis", Composite Structures, Vol. 34, pp. 29-42, 1996. 
[14]Hua, H., Sol, H., De Wilde, W.P., "On a statistical optimization method used in finite element model updating", Journal of Sound and Vibration, Vol. 231(4), pp.1071-1078, 2000

[15] Daghia, F., De Miranda, S., Ubertini, F., Viola, E., "Estimation of elastic constants of thick laminated plates within a Bayesian framework", Composite Structures, Vol. 80, pp. 461-473, 2007

[16] Pedersen, P. and Frederiksen, P.S, "Identification of Orthotropic Material Moduli by a Combined Experimental/Numerical Approach", Measurement, Vol. 10, pp. 113-118, 1992.

[17] Berger, J.O., Statistical decision theory and bayesian analysis, New York - Springer, 1985.

[18] Kaipio, J. and Somersalo, E., Statistical and Computational Inverse Problems, New York - Springer, 2005.

[19] Myers, R.H. and Montgomery, D.C., Response Surface Methodology: Process and Product Optimization Using Designed Experiments, John Wiley \& Sons, $2^{\text {nd }}$ edition, 2002.

[20] Gürdal, Z., Haftka, R.T. and Hajela, P., Design and Optimization of Laminated Composite Materials, New York - Wiley Interscience, 1998.

[21] Dickinson, S.M., "The buckling and frequency of flexural vibration of rectangular isotropic and orthotropic plates using Rayleigh's method", Journal of Sound and Vibration, Vol. 61, No. 1, pp. 1-8, 1978.

[22] Viana, F.A.C. and Goel, T., Surrogates ToolBox 1.1, http://fchegury.googlepages.com/surrogatestoolbox, 2008.

[23] Gogu, C., Haftka, R. T., Le Riche, R., Molimard, J., Vautrin, A. and Sankar, B. V., "Comparison between the basic least squares and the Bayesian approach for elastic constants identification", Journal of Physics: Conference Series, Vol. 135, No. 012045, 2008. 